\title{
Memoria y prácticas docentes: enseñanza de la violencia política peruana en un colegio público-emblemático peruano
}

Gabriela Arrunátegui

$\overline{\text { Pontificia Universidad Católica del Perú (PUCP) }}$

g.arrunategui@pucp.edu.pe

Recibido: $17 / 08 / 2020$

Aprobado: 01/07/2021 


\title{
Memoria y prácticas docentes: enseñanza de la violencia política peruana en un colegio público-emblemático peruano
}

\section{Resumen}

El objetivo de este estudio es identificar y comprender la manera en que los docentes de los cursos de Historia y Formación Ciudadana enseñan el período de violencia política peruano en un colegio público-emblemático de Lima, Perú. La metodología es cualitativa y de orientación etnográfica, mediante el empleo de observaciones de clases, entrevistas a profundidad y revisión de documentos. Para su análisis, los enfoques teóricos seleccionados fueron la sociología de la experiencia escolar, la memoria e historiografía. Los resultados ${ }^{1}$ señalan que la enseñanza de la memoria está atravesada por conflictos y negociaciones en torno al contexto institucional y político, la dinámica educativa en el aula, y las trayectorias profesionales y personales de los docentes.

Palabras clave: Educación ciudadana, docentes de secundaria, memoria colectiva, historia contemporánea

\section{Memory and Teaching Practices: The Teaching of Peruvian Political Violence in A Public-Emblematic Peruvian School}

\begin{abstract}
This study analyses the way in which the teachers of the History and Citizen Training courses teach the period of Peruvian political violence in a public-emblematic school in Lima, Peru. The methodology is qualitative and ethnographicoriented, using class observations, in-depth interviews, and document review. For its analysis, the selected theoretical approaches were the sociology of school experience, social memory, and historiography. The results indicate that the teaching of memory is intersected by conflicts and negotiations regarding the institutional and political context, the educational dynamics in the classroom, and the professional and personal trajectories of the teachers.

Keywords: Civic education, secondary school teachers, collective memory, contemporary history
\end{abstract}

1. Los resultados que se presentan a continuación forman parte de una tesis de licenciatura que analizó esta problemática de manera más amplia. 


\section{Introducción}

Entre los años 1980 y 2000, el Perú vivió el período de violencia política más intenso y prolongado de su historia republicana, con un aproximado de $69 \mathrm{mil}$ personas muertas y desaparecidas, de las cuales fueron responsables el Partido Comunista Peruano Sendero Luminoso (PCP- SL) (del 54\%) y los agentes del Estado (del 37\%). La gran mayoría de las víctimas tenía como idioma materno el quechua u otras lenguas nativas (75\%), vivía en zonas rurales $(79 \%)$ y tenía un nivel educativo inferior al secundario (68\%) (CVR, 2003). Esta investigación empleará el término de violencia política para nombrar los hechos de violencia ocurridos entre los años 1980 y 2000 en el Perú, pues, de acuerdo con Francesca Denegri y Alexandra Hibbett (2016), este visibiliza la violencia estructural del sistema político peruano que dio lugar a la guerra. Es decir, da cuenta de las realidades de un país con una larga tradición de gobiernos autoritarios interrumpidos parcialmente por fuerzas democratizantes y con una política nacional que ha permitido la imposición de unos sectores frente a otros (Denegri y Hibbett, 2016).

Si bien la violencia atravesó todas las esferas de la vida social, es difícil encontrar otro escenario institucional en el que la batalla cotidiana haya sido tan intensa como en la educativa (Cáceres, 2004). Esto se debe a que el PCP-SL empleó el sistema educativo para transmitir su ideología ${ }^{2}$, e inició sus acciones con un grupo de docentes universitarios y de escuela (Degregori, 1990). Como consecuencia, los docentes fueron estigmatizados por los gobiernos presidenciales de turno y reprimidos por las Fuerzas Armadas (FF.AA.) (Burt, 2011), mientras que, para la sociedad peruana, la figura del maestro se vio desvalorizada (Ayllón, 2012). Posteriormente, aquellos docentes que se rebelaron contra el PCP-SL también fueron reprimidos por los mismos (Degregori, 2011). Fue en este marco de violencia y silencio sistemáticos que los docentes tuvieron que desempeñar su profesión (Trinidad, 2004).

No fue hasta la transición democrática liderada por el Presidente Valentín Paniagua (2000-2001) que se buscó reconstruir el Estado-nación. Así, en el año 2001, se creó el Foro del Acuerdo Nacional ${ }^{3}$ con el objetivo de crear 31 políticas de Estado que devolvieran la estabilidad al país. A la par, se estableció la Comisión de la Verdad y Reconciliación (CVR) con el fin de investigar las violaciones a los derechos humanos durante el período de violencia política. En el Acuerdo Nacional (2011) e Informe Final de la CVR (2003), la institución educativa es seleccionada como la responsable de transmitir la memoria histórica de este pasado reciente desde un enfoque democrático y de cultura de

2. La ideología del PCP-SL es el resultado de una depuración dogmática entre marxismo, leninismo y maoísmo; y la incorporación del "pensamiento Gonzalo", en honor a su líder Abimael Guzmán. Esta ideología buscaba movilizar la lucha popular del campo a la ciudad desde un enfoque dogmático y acrítico (CVR, 2003).

3. En este, participaron representantes del gobierno, partidos políticos y sociedad civil. 
paz. Específicamente, el Informe Final recomendó al Estado reformar el sistema educativo a través de la creación de currículos nacionales que promuevan nuevos aprendizajes a través de contenidos y metodologías crítico-reflexivas; asimismo, exhortó la difusión de los hallazgos de este informe con el fin de propiciar una formación ciudadana democrática (CVR, Tomo IX, 2003). Después de aproximadamente veinte años, cabe preguntarse por la implementación de dichas políticas y recomendaciones en la escuela peruana.

Como primer hito, encontramos la creación de la Ley General de Educación (Ley N²8044) del año 2003, que coloca a la persona como centro del proceso educativo, y busca consolidar la creación de currículos básicos y articulados que impulsen la convivencia social y el ejercicio ciudadano (art. 33, p.13). En el ámbito curricular, en el primer Diseño Curricular Nacional de Educación Básica Regular del año 2005 y en su nueva versión del año 2009, el período de violencia política era uno de los temas oficiales a ser enseñado en los cursos de Formación Cívica y Ciudadana (FCC) e Historia en quinto de secundaria, así como en el actual Currículo Nacional de Educación Básica (2016). En este contexto, también se intentó incorporar los contenidos del Informe Final y la guía metodológica para docentes de cuarto y quinto de secundaria elaborados por la CVR, aunque sin mucho éxito, debido al rechazo por parte de actores políticos y FF.AA. hacia los hallazgos presentados (Jave, 2014). Así, la llegada de la historia reciente y la memoria a la escuela ha sido (y es) compleja, por lo que estos documentos curriculares no son un producto imparcial, sino el resultado de luchas por la memoria (González, 2012). La investigación de Lucía Fernández (2015), en que analiza los textos escolares peruanos de Ciencias Sociales de quinto de secundaria, es prueba de ello. La autora encuentra que dichos textos han sido el resultado de intereses, subjetividades e interpretaciones que se gestaron entre los altos cargos.

Ahora bien, el entendimiento de este fenómeno no puede reducirse a decisiones político-educativas, sino que debe examinarse qué sucede con los docentes. Desde el campo de las Ciencias Sociales y Ciencias Humanas - aunque no desde la Sociología, sí desde la Antropología, la Historia, la Psicología y la Lingüística一, se ha buscado comprender qué ocurre con los docentes. ¿Enseñan este período en las aulas? La primera en dar cuenta sobre ello fue la antropóloga peruana Rocío Trinidad (2004), quien, a través de un estudio etnográfico en las aulas entre los años 2001 y 2002, halló que, si bien los docentes de Ayacucho se reconocen como transmisores de memoria sobre este período, no siempre actúan como tales, debido a la falta de claridad curricular para abordarlo, y al temor de confrontarse con sus propios recuerdos y de ser acusados por apología al terrorismo por las autoridades educativas.

Estudios posteriores -Flores (2016), Jave (2014), Salas (2019), Uccelli et al. (2017), Valle y Frisancho (2015) - siguen dando cuenta que, pese a que los docentes reconocen la importancia de enseñar este período en sus clases, aún llevan consigo los temores que mencionaba Trinidad (2003). Estas investigaciones se han aproximado a los actores educativos a través de entrevistas, 
así como mediante talleres interactivos orientados a docentes y estudiantes de colegios y universidades. Asimismo, encuentran que aquellos docentes que señalan que sí abordan el tema en sus clases, ya sea porque lo consideran relevante o porque es lo establecido en los dispositivos curriculares del Ministerio de Educación (Minedu), no dejan de sentir los mismos temores que aquellos que no lo hacen. Por ello, como un mecanismo de protección, han optado por enseñarlo desde la "neutralidad". Es decir, pese a que en clases cuentan algunas de sus vivencias, están buscan no alejarse de la versión oficial de los libros escolares y, así, evitar generar conflicto entre sus estudiantes (Uccelli et al., 2017).

En esa línea, esta investigación sociológica se suma a lo trabajado en el Perú con un aporte particular: el análisis de las prácticas y discursos docentes en el aula al momento de enseñar este período. Se espera que los hallazgos presentados contribuyan a imaginar alternativas para trabajar en la escuela el período de violencia política en un marco de cultura de paz y enfoque de ciudadanía crítica, a través del reconocimiento de las diversas memorias de docentes y estudiantes que convergen en el espacio educativo, y que muchas veces se encuentran en disputa porque dichos actores no cuentan con herramientas pedagógicas para abordar y hacer dialogar la diversas de miradas sobre lo que ocurrió en el país.

\section{Marco teórico}

La sociología de la experiencia escolar y los estudios de memoria e historiografía fueron los enfoques empleados para analizar la manera en que los docentes de FCC e Historia enseñan el período de violencia política. La sociología de la experiencia escolar propuesta por Francois Dubet y Danilo Martuccelli (1997) permite mostrar el contexto actual en el que se encuentran la escuela y sus actores. Específicamente, contribuye a entender que el docente es un sujeto que experimenta su profesión, es decir, que construye una identidad y un sentido en el sistema educativo. De este modo, lo vemos como un agente y no alguien que solo ha interiorizado un rol. Por ello, el oficio del docente no consistirá únicamente en aplicar reglas y normas de un estatuto, porque este no solo le permite dar clases, sino que habrá un sentido de apropiación y reinterpretación de contenidos y normas educativas (Dubet y Martuccelli, 1997).

A su vez, los docentes deberán enfrentar diversas pruebas. Una de ellas es el establecimiento incierto: conjunto de programas, políticas y proyectos educativos que regulan la actividad docente que muchas veces los dejan sin capacidades reales de autonomía. Otra es la clase frágil, caracterizada por esta heterogeneidad que acompaña a los estudiantes y sus diversas maneras de aprender y adquirir competencias, producto de la masificación educativa. Los docentes, entonces, vivirán su experiencia escolar en un marco de tensiones y negociaciones entre lo oficial y sus propias experiencias.

En esa línea, al emplear el enfoque de la sociología de la experiencia escolar, esta investigación busca, en primer lugar, posicionar a los docentes como 
sujetos que se apropian y reinterpretan los contenidos oficiales de acuerdo con sus concepciones pedagógicas, sus memorias y experiencias de períodos históricos - como el de la violencia política peruana- sin dejar de reconocer los grandes retos que enfrenta su profesión. En segundo lugar, se busca rescatar la complementariedad entre los campos de la memoria e historiografía para aproximarse a pasados conflictos y recientes. La memoria es fundamental para la historiografía, porque permite plantear preguntas abiertas, creativas y productivas para la investigación y reflexión, mientras que la historiografía permite dotar de objetividad los procesos sociales y políticos.

En el campo de memoria, se emplea el término de memoria colectiva propuesta por Maurice Halbwachs (2004), quien señala que "nuestros recuerdos siguen siendo colectivos, y son los demás quienes nos los recuerdan, a pesar de que se trate de hechos en los que hemos estado implicados nosotros solos, y objetos que hemos visto solos" (p. 118). Es decir, si bien nuestros recuerdos pueden ser únicos y singulares, estos se activan en "marcos sociales", en los que las memorias (siempre plurales) encarnan valores, códigos culturales, necesidades y recuerdos que una sociedad comparte con sus miembros, y que, al transmitirlos, activan y reconstruyen nuestros recuerdos, incluso después de que hayan desaparecido. Ese es el poder de los marcos sociales: activar y reforzar las memorias (individuales) del pasado en el presente a través de un proceso de interacción con los otros.

Elizabeth Jelin (2012) señala que estas memorias se encuentran en contraposición o aún en conflicto con otras, pues cada una busca mostrar su propia, verdad y luchar por hegemonizarla o mantenerla viva. Así, surgen las luchas por la memoria (Jelin, 2012) entre aquellas que son oficiales, hegemónicas, contrahegemónicas y subterráneas. La primera se expresa a través de relatos nacionales que refuerzan sentimientos de cohesión social; y, en la escuela, se cristaliza en textos escolares (Jelin, 2012). La segunda es aquella que predomina con mayor fuerza y aceptación en diversos grupos sociales, especialmente, en aquellos con poder (Pollak, 2006). Cabe mencionar que la memoria oficial y la hegemónica no necesariamente suelen coincidir. La tercera busca resistir a la oficial y/o hegemónica por medio de relatos de transmisión oral o prácticas que ofrecen diferentes narrativas del pasado y que pueden llegar a amenazar el consenso nacional (Jelin, 2012). Finalmente, la memoria subterránea (Pollak, 2006) pertenece a culturas minoritarias y dominadas, y es trasmitida informalmente por ser considerada "prohibida".

En el caso peruano, coexisten diversas memorias que buscan posicionarse oficial y hegemónicamente dentro de la esfera pública y, por ende, en la escuela. La primera es la memoria salvadora. Esta es empleada por Steve Stein (2013) para visibilizar la narrativa que justifica la violación de los derechos humanos por parte de los militares chilenos en 1973, al otorgarles un papel salvador al frenar una posible guerra civil sin importar los medios utilizados. Degregori (1990), y, posteriormente, Barrantes y Peña (2006) emplean la memoria salva86 I dora (construida desde 1992) para mostrar que, en la sociedad peruana, se ha 
instaurado una narrativa que busca justificar las acciones autoritarias y violación de derechos humanos de Fujimori ${ }^{4}$, en la medida que se asume que este salvó al Perú de la amenaza subversiva. La segunda es la memoria para la reconciliación, empleada por Barrantes y Peña (2006) a partir de la memoria ejemplar, planteada por Tzvetan Todorov (2000). Esta memoria utiliza los hechos del pasado como lecciones de injusticias sufridas para luchar contra aquellas que se mantienen vigentes y buscar justicia en el presente. Así, con la transición democrática, se respaldó la memoria para la reconciliación propuesta por la CVR (2003), cuyos objetivos eran cinco: (1) analizar las condiciones políticas, sociales y culturales que contribuyeron a la situación de violencia, tanto desde el Estado como desde la sociedad; (2) contribuir a la administración de justicia; (3) procurar la determinación del paradero, identificación y situación de las víctimas, y, en lo posible, determinar las responsabilidades correspondientes; (4) formular propuestas de reparación moral y material de las víctimas y/o familiares; y (5) recomendar reformas para que esta situación no se repita. Finalmente, la memoria heroica, acuñada por Steve Stein (2006), engloba una serie relatos que entienden el golpe militar chileno como un acto heroico y necesario.

El Ejército peruano, a través de su libro En honor a la verdad (2012), reconoce que existe una responsabilidad compartida del Estado y las FF.AA. No obstante, también se ha podido encontrar otros de sus documentos, como el libro El terrorismo en el Perú (2003), que fue publicado en respuesta al Informe Final de la CVR (2003) y que busca confrontarla directamente para demostrar que las FF.AA. no violaron derechos humanos, sino que combatieron heroicamente el terrorismo. Se observa, entonces, que incluso dentro de las FF.AA. conviven narrativas con diversos matices sobre su participación en el período de violencia política.

Por su parte, la historia escolar (Carretero, Rosa y Gonzales, 2006) ha estado más ligada a compromisos emotivos y nacionales que a la creación del pensamiento crítico y reflexivo del pasado. El emplear la historiografía en la historia escolar evita que esta última se convierta en una sirvienta de la memoria nacionalista y la memoria colectiva esencialista (Pérotin-Dumon, 2007). De este modo, la enseñanza de una historia homogeneizadora, acrítica y memorística es reemplazada por una que se aproxima al pasado de forma compleja, comprende la causalidad histórica, emplea diversas fuentes, entre otras características disciplinares. Esto le brinda al docente herramientas para enfrentar procesos históricos recientes y complejos en los cuales las memorias de los estudiantes y de los mismos docentes pueden encontrarse en tensión.

4. Alberto Fujimori fue el presidente del Perú entre los años 1990-2000. 


\section{Metodología}

La presente investigación se enmarca en una perspectiva cualitativa y emplea los estudios de caso para analizar a profundidad y en un tiempo rigurosamente definido (Schaub y Zenke, 2001) las percepciones y prácticas de los docentes al enseñar el período de violencia política en las aulas. En esa línea, los casos seleccionados fueron docentes que deberían cumplir con los siguientes criterios:

- Ser de la especialidad de Ciencias Sociales (CC.SS.) para el nivel de educación secundaria

- Contar con más de veinte años de trayectoria profesional en este campo

- Dictar los cursos de Formación Cívica y Ciudadana (FCC) e Historia, Geografía y Economía (Historia) para el grado de quinto de secundaria

- Ser nombrados en un colegio público-emblemático que cuente con el programa Jornada Escolar Completa para el nivel de educación secundaria ${ }^{5}$

- Haber vivido y experimentado el proceso de los veinte años de la violencia política peruana (1980-2000)

Así, se seleccionaron a los cuatro docentes que enseñaban los cursos de FCC e Historia en todas las secciones de quinto de secundaria del colegio público y emblemático San Martín: Sol, Juan, Glenda y Ana ${ }^{6}$. Este colegio fue seleccionado porque, al ser emblemático, tiene una larga trayectoria histórica desde 1940. Es decir, estuvo activo durante los años de la violencia (1980-2000), trabaja con docentes de larga trayectoria profesional y alberga a una gran cantidad de estudiantes. Durante el trabajo de campo, acogía a 2517 alumnos, 159 docentes e impartía los niveles educativos de inicial, primaria y secundaria (Escale, 2017).

El trabajo de campo se realizó, desde una perspectiva etnográfica, entre los meses de setiembre y diciembre del año $2017^{7}$. Se observaron principalmente

5. Se seleccionó un colegio público con Jornada Escolar Completa (JEC) por contar con recursos didácticos y orientaciones pedagógicas para trabajar temas como el período de violencia política peruano de los años 1980-2000. Asimismo, los colegios JEC amplían sus horas pedagógicas de 35 a 45 horas a la semana, e incentivan a que todos sus docentes participen de los programas de capacitación que brinda el Minedu y reciban capacitaciones por áreas temáticas con la finalidad de mejorar los aprendizajes. En ese sentido, los docentes seleccionados parten de un marco referencial especial para trabajar el período de violencia política.

6. Se utiliza seudónimos para proteger la identidad de la institución educativa y del grupo de docentes.

7. Si bien el trabajo de campo de esta investigación se realizó en el año 2017, en el año 2016, entre los meses de mayo-junio, se llevó a cabo una primera aproximación en el mismo colegio. Asimismo, se parte de una experiencia previa: en el mes octubre del año 2016, en un colegio público de la ciudad de Huamanga-Ayacucho, se realizó entrevistas a docentes de quinto de secundaria de Ciencias Sociales y una observación en el aula. Sin embargo, el trabajo de campo no pudo continuar, debido a que la 
dos espacios del colegio: el salón del área de Ciencias Sociales (donde los docentes se reunían luego de clases) y cuatro aulas de clases de quinto de secundaria. En el primer espacio, se entabló conversaciones informales y entrevistas a profundidad que permitieron generar lazos de confianza. En las aulas, se observaron más de 40 horas de clases, de las cuales 20 estuvieron dirigidas a la enseñanza de la violencia política: 12 horas de la unidad del libro Convivencia democrática y cultura de paz de la Editorial Santillana (2013) del curso de FCC, y 8 horas en la unidad del libro Un periodo de transformaciones sociales de la editorial Santillana (2015) del curso de Historia, tal como se muestra en Tabla 1. Se decidió observar clases previas al tema del período de violencia política con el objetivo de que los docentes se sintieran cómodos al momento de enseñarlo y lo hicieran de la forma más natural posible.

Tabla 1

Observaciones de clases sobre el periodo de violencia política (1980-2000)

\begin{tabular}{|c|c|c|c|c|c|}
\hline Fecha & Aula & Tiempo & $\begin{array}{l}\text { Unidad } \\
\text { del libro }\end{array}$ & Tema & Docente \\
\hline \multicolumn{6}{|c|}{ Observaciones del curso Formación Cívica y Ciudadana en las aulas } \\
\hline $\begin{array}{c}15 / 11 / \\
2017\end{array}$ & $\begin{array}{c}\text { 5to } \\
\mathrm{H}\end{array}$ & 3 horas & \multirow{4}{*}{$\begin{array}{l}\text { Convivencia } \\
\text { democrática y } \\
\text { cultura de paz }\end{array}$} & 2.1. Convivencia democrática & Juan \\
\hline $\begin{array}{l}22 / 11 / \\
2017\end{array}$ & $\begin{array}{c}5 \text { to } \\
\text { A }\end{array}$ & 3 horas & & 2.2. El Acuerdo Nacional & \multirow{3}{*}{ Sol } \\
\hline $\begin{array}{c}29 / 11 / \\
2017\end{array}$ & $\begin{array}{c}5 \text { to } \\
\text { A }\end{array}$ & 3 horas & & $\begin{array}{l}\text { 2.3. Mecanismos democráticos } \\
\text { de resolución de conflictos }\end{array}$ & \\
\hline $\begin{array}{c}27 / 11 / \\
2017\end{array}$ & $\begin{array}{c}5 \text { to } \\
\mathrm{B}\end{array}$ & 3 horas & & $\begin{array}{l}\text { 2.4. Un pasado doloroso: la } \\
\text { crisis de violencia política en } \\
\text { el Perú }\end{array}$ & \\
\hline \multicolumn{6}{|c|}{ Observaciones del curso de Historia, Geografía y Economía en las aulas } \\
\hline $\begin{array}{c}22 / 11 / \\
2017\end{array}$ & $\begin{array}{c}\text { 5to } \\
\mathrm{F}\end{array}$ & 2 horas & \multirow{4}{*}{$\begin{array}{l}\text { Un periodo de } \\
\text { transformaciones } \\
\text { sociales }\end{array}$} & $\begin{array}{l}\text { 4.8 El regreso de la democracia- } \\
\text { El segundo gobierno de } \\
\text { Belaunde }\end{array}$ & \multirow[t]{2}{*}{ Glenda } \\
\hline $\begin{array}{c}06 / 12 / \\
2017\end{array}$ & $\begin{array}{c}5 \text { to } \\
\mathrm{F}\end{array}$ & 2 horas & & 5.9. La década del fujimorismo & \\
\hline $\begin{array}{c}22 / 11 / \\
2017\end{array}$ & $\begin{array}{c}5 \text { to } \\
\mathrm{A}\end{array}$ & 2 horas & & $\begin{array}{l}\text { 4.8 El regreso de la democracia- } \\
\text { El segundo gobierno de } \\
\text { Belaunde }\end{array}$ & \multirow[t]{2}{*}{ Ana } \\
\hline $\begin{array}{c}06 / 11 / \\
2017\end{array}$ & $\begin{array}{c}\text { 5to } \\
\text { A }\end{array}$ & 2 horas & & 5.9. La década del fujimorismo & \\
\hline
\end{tabular}

Fuente: Elaboración propia.

huelga de maestros del año 2016 lo impidió. Estas experiencias permitieron afinar los instrumentos de recojo de información. 
Asimismo, se realizaron siete entrevistas: cuatro a los docentes seleccionados y tres a diferentes autoridades escolares (director, subdirector y coordinadora del área de CC.SS). Para el primer grupo, se llevaron a cabo entrevistas a profundidad que duraron aproximadamente cinco horas; $y$, para el segundo, entrevistas semiestructuradas de aproximadamente dos horas. Finalmente, se empleó la revisión documental de informes oficiales del Minedu y la IE San Martín, así como de los docentes seleccionados. La triangulación de esas tres herramientas (Stake, 1998) permitió alcanzar mayor validez en el análisis de los resultados obtenidos.

\section{Hallazgos}

\section{Contenidos oficiales en los libros escolares}

Como se mencionó inicialmente, este artículo forma parte de una investigación más amplia sobre la enseñanza de la violencia política. En esta se analizó, además, los libros escolares de FCC e Historia de quinto de secundaria de la Editorial Santillana de los años 2013 y 2015 respectivamente, con el objetivo de enmarcar el discurso oficial que los docentes debían manejar. En esta sección, se realizará un breve resumen sobre cuáles eran esos discursos.

En primer lugar, no se encontró una sola manera de nombrar al período. El título predominante en ambos textos es "crisis de la violencia" o "violencia política". Cuando se mencionan las acciones que realizaron los grupos subversivos, se emplea el término "terrorismo"; y, cuando se menciona a la CVR, "conflicto armado interno". En segundo lugar, no se explican las causas que originaron la violencia política de los años 1980-2000. Por el contrario, pareciera que confunden las causas con el primer ataque del PCP-SL: la quema de ánforas en Chuschi, Ayacucho en 1980.

En tercer lugar, en ambos libros, este período histórico es explicado a través de los gobiernos presidenciales de Belaunde (1980-1985), García (19851990) y Fujimori (1990-2000), que abarcaron los 20 años de violencia. Los gobiernos de Belaunde y Fujimori son acusados de haber cometido violaciones a los derechos humanos, mientras que al de García de "inconsistencia de la estrategia subversiva”. Además, las capturas de los líderes del PCP-SL y el Movimiento Revolucionario Túpac Amaru (MRTA) son atribuidas a fuerzas especiales de la policía y no a un presidente en particular. Finalmente, la CVR y su Informe Final están presentes en ambos libros. En el de FCC, se emplea extractos de dicho informe, principalmente, las recomendaciones y aquellas secciones que visibilizan que la población indígena y pobre fue la más afectada. En el libro de Historia, en cambio, se muestra información que explica que la violencia afectó también a militares y a policías.

Muchos de los contenidos oficiales sobre este período aún no están claros. Por el contrario, son el resultado de luchas por la memoria (Gonzales, 90 I 2012), sobre todo en un país como el Perú (Fernández, 2015), en donde aún 
no existen consensos a nivel nacional sobre lo que se desea recordar u olvidar colectivamente.

\section{Los docentes: caracterización, memorias y opiniones sobre la enseñanza de la violencia política peruana}

En esta sección, se evidenciará que cada uno de los cuatro docentes cuenta con una historia directa y personal durante los años de la violencia, principalmente cuando eran estudiantes universitarios y en sus inicios como maestros de escuela. Además, se mostrará cuáles son sus opiniones en torno a la enseñanza de este período en la escuela.

Glenda y Juan estudiaron en la Universidad Inca Garcilaso de la Vega y La Cantuta entre 1973 y 1980, respectivamente. Durante esos años, compartieron espacios con compañeros de izquierda que pertenecían a grupos como el PCP-SL y MRTA. Mientras, Sol, quien estudió en la Universidad San Martín en $1988^{8}$, convivió con infiltrados de las Fuerzas Armadas. Ana, en cambio, estudió como primera carrera Ingeniería Agrónoma en la Universidad Nacional de San Antonio Abad del Cusco, en 1980. No presenció directamente la violencia política hasta que comenzó la carrera de Educación en Ciencias Sociales en la Universidad Nacional Hermilio Valdizán de Huánuco en 1993.

En los inicios de sus carreras, los docentes tomaron caminos distintos. Glenda y Sol enseñaron en colegios de Lima. Glenda enfrentó la muerte de uno de sus estudiantes a manos del PCP-SL, mientras que Sol señala haber trabajado en un colegio supervisado por un grupo del servicio de inteligencia militar. Juan y Ana se desempeñaron como docentes en provincia y enfrentaron el reto de las escuelas rurales. Ambos tuvieron que trabajar en condiciones precarias y enseñar a estudiantes con problemas económicos.

Cuando se les preguntó sobre la enseñanza del período de violencia política en la escuela, los docentes señalaron que, tanto en FCC como en Historia, el tema debe ser trabajado como un "hecho histórico" y sin valoraciones de por medio. Esto significa para ellos lo que ya mencionaban Valle y Frisancho (2015): emplear información oficial para así evitar exponer sus propias ideas por temor a ser acusados de apología al terrorismo. No obstante, como señala Trinidad (2003), los docentes se consideran transmisores de memoria a pesar de no hacerlo en la escuela, y el inicio de la transición democrática traería una nueva oportunidad para hacerlo, como se muestra a continuación:

8. La CVR distinguió cinco momentos en la violencia política. El primer momento llamado "El inicio de la violencia armada" comienza con la primera acción realizada por SL en Chuschi el 17 de mayo de 1980. Entonces, se infiere que entre los años que estudiaron Glenda y Juan todavía no había un enfrentamiento directo, por lo que se podía hablar de forma abierta de la lucha popular. Mientras, entre junio de 1986 y marzo de 1989, se da lo que la CVR ha nombrado como "El despliegue nacional de la violencia". En este período, en que era peligroso opinar sobre la violencia política, Sol realizaba sus estudios universitarios. 
Muy someramente, muy poco. No podemos profundizar tampoco. No podemos decir qué es la ideología y quiénes son Mao o Lenin, porque hay temor, el terrorismo viene y se los lleva [silencio] Que aprendan en el superior, cuando estén más grandes. (Juan, 2017)

Yo lo enseño de acuerdo a mi vivencia y de acuerdo a lo que sé. Uno ha sido parte de ese momento. Hay que trabajar con las emociones, con películas, para que ellos no vuelvan a caer, que sientan cómo lo vivimos. (Sol, 2017)

Causa bastante tristeza que, como la juventud no ha vivido esa realidad, tenga ideas bien equivocadas sobre lo que ha sucedido en la época más difícil del país. Por eso, hay que enseñar con crudeza; solo así aprenderán. (Glenda, 2017)

Hay que enseñarles a los estudiantes la realidad cruda que se vivió en esos años. Ellos tienen que asumir la responsabilidad social, porque la historia no es que se detenga, la historia continúa y hay consecuencias ahorita. (Ana, 2017)

Según los testimonios, existe un temor a que se repita una situación similar en el país. Para evitarlo, Glenda, Ana y Sol señalan que el tema debe ser enseñado de manera potente a las nuevas generaciones, mientras que, para Juan, habría que hacerlo con mucho cuidado, apelando a poca información y sin profundizar en ideologías. Estas respuestas son el reflejo de un pasado que no es fácil de enseñar, porque aún se encuentra presente en las historias de vida de los docentes, tal como ya han dado cuenta las investigaciones de Flores (2016), Jave (2014), Salas (2019), Trinidad (2003), Uccelli et al. (2017), y Valle y Frisancho (2015). A continuación, se observará cómo es que, a pesar de señalar que quieren trabajarlo en clases desde la "neutralidad", las creencias, ideales y posturas de los docentes sobre lo ocurrido durante esos años impregnan sus discursos y sus prácticas.

\section{Prácticas y discursos en torno a la enseñanza de la violencia política en las aulas}

El conflicto que puede generar la historia reciente de un país se intensifica cuando sus distintas visiones se encuentran en el aula (Carretero y Borrelli, 2008). Frente a ello, el análisis se dará en función de los temas que más trabajaron los docentes en las clases observadas: (1) introducción del período; (2) la manera de nombrarlo; (3) las causas; (4) los actores más mencionados: grupos subversivos, Fuerzas Armadas y gobiernos presidenciales de turno; y (5) la Comisión de la Verdad y Reconciliación y su Informe Final. A través de estos temas, se identifican qué herramientas curriculares y pedagógicas emplean y cómo se apropian de ellas, así como los relatos del pasado reciente que transmiten en el aula y su relación con las memorias públicas. 
Introducir el tema en las clases

La introducción del período en el aula depende del área curricular. En FCC, trabajaron este período como un tema particular. Sol inició la clase con la presentación de la sesión de aprendizaje "Rechazando todo tipo de violencia" y les explicó a sus estudiantes las competencias y las capacidades que deseaba desarrollar. Juan, por su parte, lo hizo mediante una lectura titulada "Los frutos amargos de la guerra", que se encuentra en el libro oficial de FCC (2013). Juan, a diferencia de Sol, logró que sus estudiantes, a partir de la lectura de una historia personal, identifiquen el período y compartan sus memorias sobre cómo los peruanos vivieron esos años. Se observa, entonces, que el recuperar la memoria colectiva en el aula permite que los estudiantes sean capaces de identificar esos acontecimientos de dolor y ubicarlos en un período que ellos denominan "terrorismo".

En el curso de Historia, la dinámica tiene otros matices. Las dos docentes decidieron enseñar el período no como un tema, sino como un subtítulo dentro de los gobiernos presidenciales de Belaunde, García y Fujimori. Esto se contradice con el esquema del libro escolar de Historia, que presenta el período como un tema en particular. Sin embargo, para ellas fue la forma más pertinente para abordarlo, como precisaron en las entrevistas. Ana indicó: "Es mejor a la antigua porque si no nunca terminamos. Son muchos temas. No nos podemos detener en todos, pero sí tocarlos de pasada en cada gobierno. Además, para que entienda el contexto en el que ocurre" (Ana, 2017). Glenda, por su parte, señaló: "No siempre uso la programación. Si pongo ese tiempo, ya no enseñaría nada, solamente el título. Yo lo hago a mi criterio. Así me llamen la atención, digan lo que me digan, así me vengan a monitorear. Yo enseño de acuerdo a mi conciencia" (Glenda, 2017).

Las docentes de Historia, Ana y Glenda, se apropiaron de la programación de la enseñanza de la violencia política, no de una forma acrítica, sino que evaluaron estratégicamente cuándo hacerlo. Esto reflejó no solo el temor de trabajar de manera profunda el período, sino también lo mencionado por Valle y Frisancho (2015): el tipo de formación pedagógica que han recibido en CC.SS. se sirve de los gobernantes como eje articulador de las narrativas históricas.

\section{Las heterogéneas y contradictorias formas de nombrar al período}

Los cuatro docentes utilizaron el término terrorismo cuando explicaron el tema oralmente, contaron anécdotas del período y dialogaron con los estudiantes. Sol, Juan y Ana, además, emplearon "Crisis de la violencia política en el Perú (1980-2000)" como título en la pizarra e indicaron a sus estudiantes que así lo escribieran en el cuaderno. Glenda, en cambio, señaló que la única forma de nombrar al período es "terrorismo" por las razones a continuación expuestas: 
Fue terrorismo porque esos son delincuentes que mataron, que querían llegar al gobierno, pero por la fuerza. Secuestraban niños, los adiestraron, así como están ahora en Siria. Inclusive, cuando vino el congresista Miyashiro, pasó un video donde un niño de más o menos 8 años agarraba del pelo a un hombre y le cortó el cuello. Eso es terror, señorita, eso no es guerra civil o un simple conflicto interno... (Glenda, 2017)

El término Conflicto Armado Interno, empleado en los libros de FCC (2013) e Historia (2015) al momento de presentar a la CVR, no fue utilizado en ninguna de las clases observadas. En las entrevistas, los docentes señalaron que era un término que se empleaba sobre todo en espacios académicos, como plantea Sol: "Eso fue hecho por académicos de la Católica, ¿verdad?... Para mí, es un término que se usa más en esos espacios" (2017).

Pareciera, entonces, que cada término se vuelve jerárquico y asignable a un grupo social, como si estos no pudiesen describir la complejidad de esos años. Como menciona Renata Bregaglio, el conflicto armado interno y el terrorismo no son excluyentes, sino que pueden nutrirse el uno del otro: "Mientras el primero hace referencia al tipo de conflictividad vivida, el segundo se refiere a los actos de violencia concretos perpetrados" (Idehpucp, 2013). Sin embargo, como lo ejemplifica el caso de Glenda, el solo llamarle al período "terrorismo" responde no a una falta de criterio, sino a las experiencias previas que cargan de significado al término.

\section{Las causas que originaron el período de violencia política}

En el curso de FCC, los docentes no trabajaron las causas que se exponen en el libro escolar de FCC (2013): expusieron contextualización temporal, geográfica e ideológica del surgimiento de la violencia política. Sol, por ejemplo, mencionó que el terrorismo inició porque el grupo terrorista Sendero Luminoso decidió quemar las ánforas en la localidad de Chuschi, departamento de Ayacucho, durante las elecciones presidenciales de 1980. Juan, docente de la Universidad La Cantuta, que convivió directamente con senderistas, expuso como causa la llegada de ideas comunistas de la Unión Soviética al Perú: "Miren, la Unión Soviética tenía ya esas ideas; mucha gente viaja allá a conocer su cultura. De ahí, nacen las ideas del comunismo que se expande a varios lugares del mundo, uno de ellos el Perú. Entonces, de ahí toman su ideología SL" (Notas de observación de clase de Juan, 2017).

En el área de Historia, las causas sí son trabajadas como parte de un proceso histórico. Sin embargo, estas se reducen a eventos políticos que no son justificados por medio de cifras o fuentes históricas, sino que responden a conocimientos que las docentes han ido adquiriendo a lo largo de su práctica profesional durante y sobre ese período. Esto se vio reflejado principalmente en el curso de Ana, como se muestra a continuación: 
Ana: Como ya les había señalado anteriormente, la Izquierda Unida había ganado algunas alcaldías en las elecciones municipales del 81 . Sin embargo, había otro grupo que se separó del Partido Comunista Peruano, como "Sendero Luminoso". Este grupo tenía otras ideas. Mientras que la izquierda que estaba en el gobierno quería el regreso de la democracia, Sendero Luminoso quería, y de hecho hizo, la revolución.

Estudiantes: ¡Ah! Y como no querían democracia quemaron las ánforas de votación, ¿no?

Ana: Exactamente. Sendero atacó en Chuschi, en el departamento de Ayacucho. Así se inició el terrorismo.

(Notas de observación de clase de Ana, 2017)

Sin embargo, al igual que en el curso de FCC, se consideró como causa principal de la violencia, la decisión del PCP-SL de quemar las ánforas en Chuschi. En ese sentido, la explicación se vuelve monocausal, pese a que las docentes de Historia tienen la posibilidad de articular las causas de este período con otros acontecimientos que se explican dentro de cada gobierno presidencial.

\section{Los actores más importantes del período}

Los docentes consideraron que los actores principales de este período son tres: grupos terroristas, FF.AA. y líderes de los gobiernos presidenciales. En primer lugar, sobre los grupos terroristas, se trabajó en clases la vida de los líderes, los atentados que cometieron y su ideología política. Los docentes emplearon información que iba más allá de la que se encontraba en los libros oficiales de FCC (2013) e Historia (2015). Al abordar el tema de los líderes, se identificó una clara preferencia en trabajar la vida de Abimael Guzmán, líder de PCP-SL, en contraposición al líder del MRTA, Víctor Polay. Los atentados cometidos por el PCP-SL y el MRTA que más se trabajaron en las clases fueron los casos emblemáticos. Por ejemplo, al PCP-SL lo vincularon con ataques en Ayacucho, principalmente, con la quema de ánforas de Chuschi; y al MRTA, con el secuestro en la Embajada de Japón. Ningún docente mencionó atentados que vayan más allá de Lima o Ayacucho. En ese sentido, se dejó de lado la posibilidad de que los estudiantes conozcan cómo el resto del país experimentó la violencia terrorista, así como la oportunidad de vincular esos hechos con los lugares de procedencias de sus padres, madres o abuelos.

$\mathrm{Al}$ mencionar en clases los atentados terroristas, los cuatro docentes inevitablemente transmitieron con nostalgia sus vivencias personales. Si bien en las entrevistas habían señalado que enseñaban desde la neutralidad, en las sesiones, se posicionaron como víctimas de esa historia reciente, debido a que el proceso pedagógico activó sus propias memorias sobre el período. Un ejemplo de ello es lo que menciona Glenda en su clase de Historia: 
¡Qué dolor habrá sentido su familia, tanta gente que ha desaparecido sin saber o muerte sin esperárselo! Yo recuerdo que estaba enseñando en el colegio y apareció en las noticias el rescate a la Embajada de Japón. Era un día cualquiera como hoy, y paramos las clases y nos pusimos a ver. (Notas de observación de clase de Glenda, 2017)

Es importante mencionar que, en los libros oficiales, no se encontró información sobre las ideologías políticas del PCP-SL y del MRTA. Sin embargo, en las observaciones, se identificó que los docentes hacían el esfuerzo por explicarlas, pero, al no contar con información oficial en el libro de texto, las enseñaban basándose en los conocimientos que han ido adquiriendo como educadores. Hablaron de "la izquierda", "el comunismo", "el socialismo" y el "capitalismo", como en el caso de Juan:

Miren, la Unión Soviética tenía ya esas ideas, mucha gente viaja allá a conocer su cultura. De ahí nacen las ideas del comunismo que se expande a varios lugares del mundo, uno de ellos el Perú, entonces, de ahí toma su ideología SL. (Notas de observación de clase de Juan, 2017).

No obstante, también se evidenció un temor por profundizar en ello, como precisó Glenda en la entrevista:

No, porque los chicos son chicos. La mente de ellos no da. Por eso es que el terrorismo agarra y a los más jóvenes se los lleva. Entonces, no se debe tocar eso. Eso se tiene que ver en todo un año. Para enseñar el materialismo dialéctico, el materialismo histórico, hay que tener capacidad. Entonces, si nosotros yendo a la universidad se nos hace difícil entender ese tipo de cosas, imagínese a los muchachos. (Glenda, 2017)

En segundo lugar, para los docentes, el hablar de los "grupos terroristas" - como los docentes llaman al PCPS-SL y MRTA - implica también recordar las acciones de la Policía Nacional del Perú y las FF.AA. De acuerdo con los libros escolares de FCC (2013) e Historia (2015), estos actores contribuyeron a devolver el orden al país, pero al mismo tiempo emplearon acciones represivas muy duras para combatir al PCP-SL y al MRTA. Pese a ello, en clases, el mensaje principal giró en torno a la heroicidad. Así, si bien se les reconoce "excesos", este discurso fue rápidamente reemplazado por mensajes como el de Glenda:

Hay que pensar ahora por nuestras Fuerzas Armadas. ¿Cuántos héroes de la patria han quedado mutilados? Otros tienen que vivir escondidos porque a veces se les responsabiliza de las muertes, solo por el hecho de haber llevado el uniforme. Gente inocente murió, pero es así en una guerra. Pero los irresponsables fueron otros, los terroristas. (Notas de observación de clase de Glenda, 2017) 
Ana, en cambio, es la única docente que mostró una posición crítica sobre las FF.AA. en sus clases. Tal como se indica en el libro de Historia (material que utilizaba constantemente), la docente sí mencionó que violaron derechos humanos. Para explicar esta idea a sus estudiantes, recurrió a sus recuerdos y experiencias:

Yo pienso que de ambas partes ha habido una criminalidad. Cuando yo estuve en esa comunidad, se llevaban su comida a balazos, hasta abusos sexuales a mujeres quechua hablantes. ¿Quién ha hecho los abusos sexuales? No son los terroristas; fue el Ejército. Yo recuerdo a los niños abandonados por los Sinchis. La gente campesina pagó la peor parte. (Notas de observación de clase de Ana, 2017)

Con respecto a la enseñanza sobre los gobiernos presidenciales y su rol durante la violencia política, durante las observaciones de clases, se notó que tanto el curso de FCC como el de Historia trabajaron este período dentro de los gobiernos de Belaunde (1980-1985), García (1985- 1990) y Fujimori (19901995 y 1995-2000). En la clase del gobierno de Belaunde, los docentes de ambas áreas señalaron que durante ese período se iniciaron las acciones terroristas del PCP-SL, las que, en un primer momento, fueron ignoradas por el presidente, pues no logró identificar la magnitud de lo acontecido. Al abordar el gobierno de Alan García, lo que más recordaron fueron los toques de qued $a^{9}$ y la formación de largas colas para que la población pueda suministrarse de alimentos, debido a la crisis económica. Si bien esta información no se encuentra en los libros de texto, son los docentes quienes decidieron transmitirla a través de recuerdos y vivencias personales, como se muestra a continuación:

Yo recuerdo que una vez mi vecina estaba enferma, tenía que ir a la posta, pero era de madrugada. Salieron con su banderita blanca, pero con mucho temor. Nosotros ya sabíamos que pasada tal hora no podíamos salir. En la casa que estuviéramos, ahí nos quedábamos. (Notas de observación de la clase de Sol, 2017)

Por último, en las clases sobre los dos gobiernos de Fujimori, todos los docentes - en las entrevistas y en clases - recalcaron que fue este presidente quien acabó con el terrorismo, y mejoró las condiciones económicas y sociales en el Perú, tal como se evidencia en la siguiente cita: "Fujimori acabó con el terrorismo. Los terroristas habían avanzado de una manera brutal, que ni el gobierno de García y Belaunde lo pudieron controlar porque ambos eran

9. El toque de queda, en el período de violencia política peruana, consistió en la prohibición a que los civiles circularan libremente por las calles de la ciudad desde las 10 pm hasta las $6 \mathrm{am}$. La medida fue establecida por instituciones gubernamentales y vigiladas por las fuerzas del orden: Policía Nacional y Ejército peruano. 
permisivos. Cuando entró Fujimori, él puso mano dura" (Ana, 2017). Asimismo, los estudiantes de la sección 5to $\mathrm{H}$, sección en la que enseña Juan, tuvieron que realizar el siguiente mural como parte de una actividad de aprendizaje:

Figura 1

Mural sobre la época de violencia política

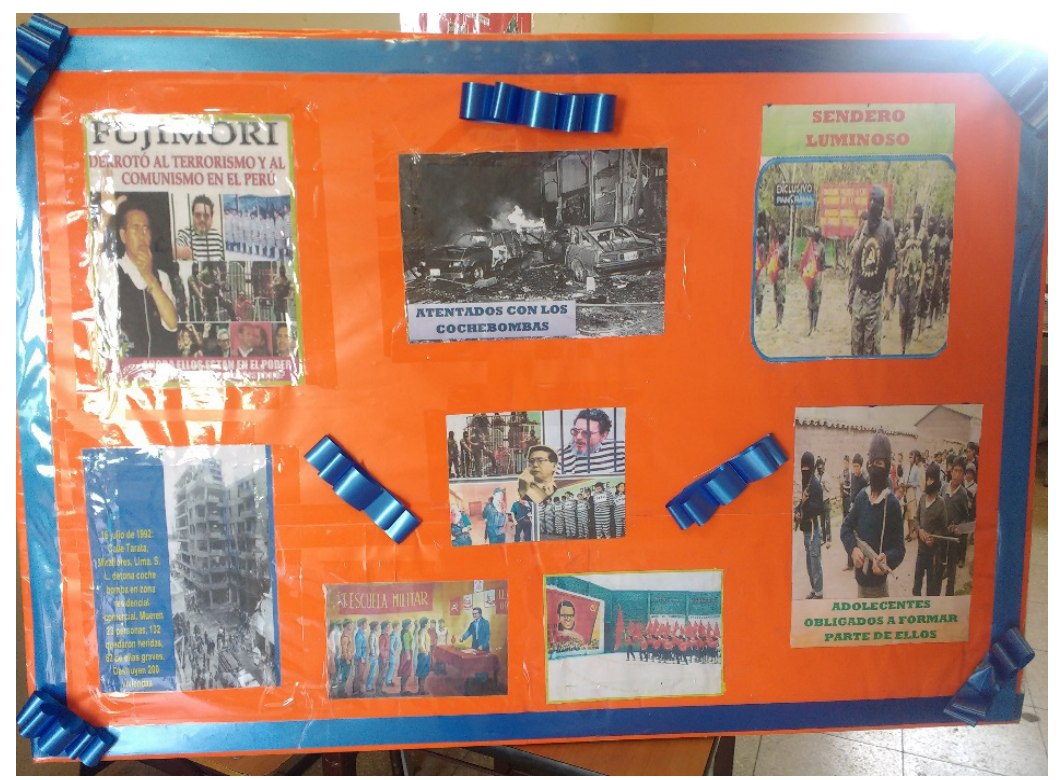

Fuente: Fotografía tomada en el salón de Juan, 2017.

En el mural, se muestra una versión simplificada sobre lo que fue el período de violencia política. En dicha versión, el PCP-SL inició las acciones armadas y el expresidente Fujimori acabó con estas a través de la captura de Abimael Guzmán.

\section{La enseñanza sobre la CVR y su Informe Final}

En el área de FCC, solo Sol mencionó a la CVR y su Informe Final a través de datos y fechas concretas, como se muestra en la siguiente observación en clases:

Sol: A ver, ya cuando se ha derrotado el terrorismo, se crea una Comisión de la Verdad y Reconciliación. ¿Alguien sabe en qué gobierno fue?

Estudiantes: ¿Qué es la CVR?

Sol: Es una comisión que recogió testimonios luego del terrorismo para saber cómo les afectó a los peruanos. Esta comisión se hizo en 
el gobierno de Paniagua y el director fue Salomón Lerner. Pero fue entregada en el gobierno de Toledo ${ }^{10}$.

(Notas de observación de la clase de Sol, 2017)

Juan, en cambio, evitó hablar de la CVR en clases porque considera que posee información sesgada. En la entrevista, el docente afirmó:

La conozco. Tengo mis dudas, porque en 1999 yo fui a trabajar lejos de Huancavelica, 2 horas de caminata. Me contaban los pobladores que había un señor que apoya al terrorismo y, cuando se fue el terrorismo, apoyó a los militares. Jugaba a los dos bandos. Cuando llegó la CVR, él dijo que fue el agraviado. Yo no puedo decir que la CVR ha sido muy estricta, muy verídica. La verdad, es que me indigna. Estaba dirigida políticamente por Toledo; quisieron dañar a Fujimori. (Juan, 2017)

Se puede observar, entonces, que, pese a que oficialmente debe enseñar sobre la CVR, su experiencia personal vivida en Huancavelica ${ }^{11}$ y su tendencia política marcan su enseñanza.

En el libro escolar de Historia (2015), también hay un apartado especial sobre la CVR. En las observaciones de clases, se notó que solo Glenda mencionó a esta institución, pese a que ella no empleaba el libro oficial de Historia (2015). Al analizar el contenido, se identificó que la información que brindó a los estudiantes era errónea. La docente señaló: "Quien presidió la CVR fue Mario Vargas Llosa y... el total de muertos que señala esta comisión [69 mil] fue solo responsabilidad de los grupos terroristas (PCP-SL y MRTA)" (Glenda, 2017), cuando en realidad el presidente de la CVR fue Salomón Lerner y el número total de víctimas se adjudica tanto al PCP-SL y al MRTA como a los agentes del Estado.

En general, los docentes saben de la existencia de la CVR, pero no todos la trabajaron en sus clases por los siguientes motivos: (1) no conocen toda la información, las posturas ni los contrastes; (2) sienten que la información brindada es sesgada, porque sus experiencias durante los años de la violencia no se ven reflejadas en el Informe Final; y (3) tienen una tendencia política propia. Cabe anotar que el Informe Final de la CVR no es un material de apoyo pedagógico en el proceso de enseñanza-aprendizaje. Es decir, no es considerado una fuente para preparar clases o resolver dudas en el aula, ni es usado como un documento de estudio para los estudiantes, como también ha evidenciado la investigación de Uccelli et al. (2017).

10. Alejandro Toledo Manrique fue presidente del Perú entre los años 2001 y 2006.

11. Este es un departamento del Perú, ubicado en la en la zona sur de la Cordillera de los Andes. 


\section{Conclusiones}

Como objetivo de esta investigación, se apuntó a identificar y comprender la manera en que los docentes de los cursos de Historia y Formación Ciudadana enseñan el período de violencia política peruano (1980-2000) en un colegio público-emblemático de Lima, Perú. A partir de este estudio, se puede señalar que los docentes enseñan dicho período en un marco de tensiones y negociaciones que se da entre la construcción de sus propias memorias sobre el tema y las exigencias de su desempeño profesional para incorporar datos oficiales que se encuentran en los libros escolares de FCC (2013) e Historia (2015). En esa línea, transmiten en sus clases relatos sobre el período de violencia política que se relacionan principalmente con la memoria salvadora; la memoria de los militares; y, en menor medida, con la memoria de la CVR. No obstante, son sus propias memorias las que finalmente refuerzan o deslegitiman alguna de ellas.

En cuanto a la enseñanza del período, se puede concluir que los cuatro docentes del colegio San Martín lo abordan a través de la apropiación y la reinterpretación de los documentos oficiales del Minedu y de su institución educativa. En ese proceso, intervienen sus concepciones pedagógicas y disciplinares de las Ciencias Sociales, sus experiencias de vida durante los años de la violencia política (como estudiantes universitarios y docentes), la cultura escolar de la institución, y las disputas públicas por hegemonizar una memoria oficial de lo ocurrido. Asimismo, sus opiniones siempre están presentes. Si bien el manual de texto da una pauta en el desempeño de la clase, no evita que los docentes se posicionen desde un rol - víctima, sobreviviente o espectador-, pues rememoran un período que han vivido directamente. A pesar de ello, el temor de ser acusados por apología al terrorismo y generar conflicto en una institución educativa que históricamente ha buscado evitarlo para construir consensos en torno a la idea de Nación genera que también lo enseñen con excesivo cuidado.

La gran mayoría de los relatos que los docentes transmiten en el aula no se encuentran plasmados en los libros escolares; son sus propias memorias y las públicas las que moldean los contenidos que transmiten. En primer lugar, se identifica que, al abordar las condiciones que hicieron posible el surgimiento de la violencia política, los cuatro docentes confunden las causas con el primer acontecimiento que marca el inicio del período, información que también se encuentra en los libros oficiales de FCC e Historia. Esto genera (1) que no enseñen las causas o que sean los docentes quienes decidan qué mencionar como causas, y (2) que la responsabilidad recaiga únicamente en el PCP-SL, explicación monocausal que invisibiliza las condiciones estructurales a nivel local, regional, nacional e internacional que influenciaron en la exacerbación de la violencia política.

En segundo lugar, vinculan el relato sobre las FF. AA y su desempeño entre 1980-2000, principalmente, con la memoria que las FF.AA. plasman en su 100 I libro En honor a la verdad (2012). Estos son los casos de Sol y Juan (docentes 
de FCC) y Glenda (docente de Historia), quienes en sus clases justifican y respaldan los "errores" cometidos por militares y policías. Mientras, Ana (docente de Historia) sí menciona en clases que las FF.AA. violaron derechos humanos, tal como se indica en el libro de Historia (material que utiliza constantemente). Para reforzar esta idea, recurre a sus recuerdos y experiencias de esos años.

En tercer lugar, cuando desarrollan las sesiones sobre los gobiernos presidenciales y cómo afrontaron la violencia política, los docentes transmiten principalmente sus propias memorias, en lugar de lo señalado en los libros oficiales de FCC e Historia. En clases, restan poder de decisión y acción sobre el incremento de la violencia a los gobiernos de Belaunde y García. Solo a Fujimori se le atribuye la derrota del "terrorismo", gracias a las capturas de los líderes del PCP-SL y el MRTA. Si bien esta premisa corresponde a la memoria salvadora, esta se ve reforzada por sus propias experiencias. Por ejemplo, todos mencionaron que presenciaron la captura del líder del PCP-SL, Abimael Guzmán, en el gobierno de Fujimori, mientras que, en los otros gobiernos presidenciales, experimentaron inseguridad y agudización de la crisis económica.

Finalmente, la versión de la CVR y su Informe Final (2003) no ha logrado ser parte de las narrativas de los docentes al momento de enseñar el período de la violencia política en las aulas. En las entrevistas, mencionaron que esto se debe a que (1) no conocen el contenido del Informe Final, (2) sus experiencias de vida no se ven reflejadas en este material y (3) la responsabilidad en la violación de Derechos Humanos que se le asigna a Alberto Fujimori va en contra de su tendencia política "fujimorista". A partir de lo observado en clases, se identificó que solo Sol (docente de FCC) y Glenda (docente de Historia) mencionaron a la CVR, aunque la primera lo hizo a través de datos y fechas acotadas; y la segunda, brindando información errónea.

\section{Recomendaciones}

A partir de los principales hallazgos, se plantean las siguientes recomendaciones al Ministerio de Educación del Perú. En primer lugar, se sugiere incentivar que la formación docente (inicial y continua) ofrezca espacios para reflexionar sobre las formas de enseñanza de la historia reciente en las áreas de Historia, Geografía y Economía, y Formación Ciudadanía y Cívica.

En segundo lugar, es preciso discutir y definir el tipo de perfil docente que se desea formar para el desarrollo de prácticas democráticas en la escuela. Si bien el actual Currículo Nacional de Educación Básica (2016) busca impulsar la construcción de un docente reflexivo, esta apuesta no será suficiente si no se implementan dispositivos y capacitaciones permanentes que estén alineadas y permitan aterrizar esta perspectiva en el aula.

En tercer lugar, se recomienda impulsar la enseñanza del período de violencia política como una oportunidad para lograr el desarrollo de competencias y capacidades del nuevo Currículo Nacional de Educación Básica (2016), como son "convive y participa democráticamente", "construye interpretaciones 
históricas" y "construye su identidad". Finalmente, se sugiere incorporar las causas de la violencia política en el aula en los libros de Historia, Geografía y Economía, y Formación Ciudadanía y Cívica.

\section{Agradecimientos}

Quisiera agradecer a mi mentora y asesora, Fanni Muñoz, por acompañarme en todo el proceso de investigación. A Sol, Glenda, Ana y Juan, por confiar en mí y permitirme escuchar sus experiencias durante la época de violencia política, por dejarme entrar a sus clases y conocer más de su trabajo. Sin ustedes, esta investigación no hubiese sido posible. 


\section{Referencias}

Ayllón Yares, G. (2013) La pedagogía memoria como elemento fundamental para la formación en derechos humanos [Tesis de Maestría, Pontificia Universidad Católica del Perú].

Asociación Defensores de la Democracia Contra el Terrorismo [ADDCOT] (2003). El terrorismo en el Perú 1980 - 2000. Versión de los militares que lo combatieron. ADDCOT.

Barrantes, R. y Peña, J. (2006). Narrativas sobre el conflicto armado interno en el Perú: la memoria en el proceso político después de la CVR. Transformaciones democráticas y memorias de la violencia en el Perú, (2), 16-40.

Burt, J. M. (2011). Violencia y autoritarismo en el Perú: bajo la sombra de Sendero y la dictadura de Fujimori. Instituto de Estudios Peruanos, SER, EPAF.

Cáceres, C. (2013) Discursos sobre reconciliación: el caso de los presos desvinculados de Sendero Luminoso y MRTA [Tesis de Maestría, Pontificia Universidad Católica del Perú].

Carretero, M., Rosa, A. y González, M. F. (2006), Enseñanza de la historia y memoria colectiva. Paidós.

Comisión Permanente de Historia del Ejército del Perú (2012). En honor a la verdad. Versión del Ejército sobre su participación en la defensa del sistema democrático contra las organizaciones terroristas. Gráfica Cánepa.

Comisión de la Verdad y Reconciliación [CVR] (2003). Comisión de la Verdad $y$ Reconciliación. Informe Final. Comisión de Entrega de la CVR.

Degregori, C. I. (1990). Ayacucho 1969-1979: El surgimiento de Sendero Luminoso del movimiento por la gratuidad de la enseñanza al inicio de la lucha armada. Instituto de Estudios Peruanos.

(2003). Jamás tan cerca arremetió lo lejos. Memoria y violencia política en el Perú. IEP y Social Science Reserch Council.

. (2011). Qué difícil es ser Dios: el Partido Comunista del Perú, Sendero Luminoso y el conflicto armado interno en el Perú: 1980-1999. Instituto de Estudios Peruanos.

Denegri, F. y Hibbett, A. (2016). Dando cuenta: estudios sobre el testimonio de la violencia política en el Perú (1980-2000). Pontificia Universidad Católica del Perú, Fondo Editorial.

Dubet, F. y Martuccelli, D. (1997). En la escuela: sociología de la experiencia escolar. Losada.

Fernández Bravo, L. (2015), La memoria en (re) construcción: las representaciones de la violencia política en el proceso educativo peruano [Tesis de Maestría, Pontificia Universidad Católica del Perú]. 
Flores Suárez, G. (2016). Creencias de profesores, padres y alumnos sobre el tratamiento en aula del conflicto armado interno peruano (1980-2000) en una institución educativa de Santa Rosa de Sacco (Yauli, Junín) [Tesis de Maestría, Pontificia Universidad Católica del Perú].

Gobierno del Perú (2002). Acuerdo Nacional. Gobierno del Perú.

Gonzales, M. P. (2012). Historia y memoria del pasado reciente en la escuela: una mirada a la propuesta oficial. Quinto Sol, 16(2), 23-37. https://cerac. unlpam.edu.ar/index.php/quintosol/article/view/31/504

Halbwachs, M. (2004). La memoria colectiva. Prensas Universitarias de Zaragoza.

Jave, I. (Coord.) (2014). Entre el estigma y el silencio: memoria de la violencia entre estudiantes de la UNMSM y la UNSCH. Instituto de Democracia y Derechos Humanos de la Pontificia Universidad Católica del Perú y Fundación Konrad Adenauer.

Jelin, E. (2012). Los trabajos de la memoria. Instituto de Estudios Peruanos.

Ley 28044 de 2003. Ley General de Educación. 29 de julio de 2003. El Peruano No. 8437.

Ministerio de Educación del Perú [Minedu] (2005). Diseño Curricular Nacional de Educación Básica Regular. Proceso de articulación. Minedu. de Educación Básica Regular. Minedu. . (2009). Diseño Curricular Nacional Educación Básica. Minedu.

(2016). Currículo Nacional de (2017). Estadísticas de la Calidad Educativa: Escale. http://escale.minedu.gob.pe/magnitudes

Pérotin-Dumon, A. (2007). Enseñar el pasado latinoamericano cercano, o el presente de la historia en Chile. En: M. Stabili (Ed.), Entre historias $y$ memorias (pp. 199-228). Vervuert Verlagsgesellschaft. https://doi. org/10.31819/9783964562609-009

Pollak, M. (2006). Memoria, olvido, silencio. La producción social de identidades frente a situaciones límite. Ediciones $\mathrm{Al}$ Margen.

Salas Franco, M. (2019). Memorias y prácticas docentes sobre el conflicto armado Interno en una IE rural en Pangoa [Tesis de Maestría, Pontificia Universidad Católica del Perú].

Schaub, H. y Zenke, K. (2001). Diccionario Akal de pedagogía. Ediciones Akal. Stake, R. (1998). Investigación con estudio de casos [Archivo en PDF]. Morata. https://www.uv.mx/rmipe/files/2017/02/Investigacion-con-estudios-decaso.pdf

Stern, S. (2006). Remembering Pinochet's Chile: On the Eve of London 1998. Duke University Press. 
Stern, S. (2013). Memorias en construcción: los retos del pasado presente en Chile, 1989-2011. Museo de la Memoria y de los Derechos Humanos.

Trinidad, R. (2004). El espacio escolar y las memorias de la guerra en Ayacucho. En: E. Jelin y F. Guillermo Lorenz (Eds.), Educación y memoria. La escuela elabora el pasado (pp. 11-39). Siglo XXI.

Todorov, T. (2000). Los abusos de la memoria. Editorial Paidós.

Uccelli, F., Agüero, J. C, Pease, M. A. y Portugal, T. (2017). Atravesar el silencio: memorias sobre el conflicto armado interno y su tratamiento en la escuela. Instituto de Estudios Peruanos.

Valle, A. y Frisancho, S. (2015). Un tema difícil de abordar Sendero Luminoso y los profesores de Ciencias Sociales en Ayacucho. En: A. Hernández, C. García y L. de la Montaña (Eds.), Una enseñanza de las ciencias sociales para el futuro: recursos para trabajar la invisibilidad de personas, lugares $y$ temáticas (pp. 1023-1029). Universidad de Extremadura, Servicio de Publicaciones. 